\title{
A Parameterized Base Station Power Model
}

\author{
Hauke Holtkamp, Gunther Auer \\ DOCOMO Euro-Labs \\ D-80687 Munich, Germany \\ Email: \{holtkamp, auer\}@docomolab-euro.com \\ Vito Giannini \\ IMEC \\ Leuven, Belgium \\ vito@imec.be \\ Harald Haas \\ Institute for Digital Communications \\ Joint Research Institute for Signal and Image Processing \\ The University of Edinburgh, EH9 3JL, Edinburgh, UK \\ E-mail: h.haas@ed.ac.uk
}

November 7, 2014

\begin{abstract}
Power models are needed to assess the power consumption of cellular base stations (BSs) on an abstract level. Currently available models are either too simplified to cover necessary aspects or overly complex. We provide a parameterized linear power model which covers the individual aspects of a BS which are relevant for a power consumption analysis, especially the transmission bandwidth and the number of radio chains. Details reflecting the underlying architecture are abstracted in favor of simplicity and applicability. We identify current power-saving techniques of cellular networks for which this model can be used. Furthermore, the parameter set of typical commercial BSs is provided and compared to the underlying complex model. The complex model is well approximated while only using a fraction of the input parameters.
\end{abstract}




\section{Introduction}

Recently, the power consumption of cellular networks has become a point of interest in research and even been taken into consideration for the standardization of future cellular networks like Long Term Evolution (LTE)Advanced [1]. It was found in 2] that in cellular networks the element which causes the largest share of overall consumption is the base station (BS). Numerous techniques have consequently been proposed by which the power consumption of BSs can be reduced [3] 8 . Some of these techniques only consider transmission power while others take into consideration that the generation of the radio signal also consumes power in circuitry by employing power models. Such power models describe abstractly how much power a transmitter consumes and how this consumption depends on operating parameters. In the past, the modelling of BS power consumption often had to be based on intuition until the first power models were published [9 13]. Simple models like [9, 10] allow computing the power consumption of a BS for specific configurations. In contrast, the non-linear complex model described by Desset et al. [13] is derived from the combination of each of a BS' subcomponents. This allows inspecting the power consumption to such detailed level as the effect of giga operations per second or transistor gate lengths, but is unwieldy to apply. In this paper, we extend the work in [11,12 by maintaining simplicity while integrating two relevant operating variables into the model, namely the power amplifier (PA)'s output range and the transmission bandwidth. The proposed model allows assessing the power consumption of all techniques that are currently employed to reduce the power consumption of BS while conserving simplicity.

The scope of the model is described in Section 2 . The model is subsequently presented in Section 3, In Section 4, it is discussed and compared to the complex model. The paper is concluded in Section 5.

\section{Power Model Scope}

Power saving techniques in literature can be generally divided into design changes and operating approaches. Design changes affect the layout of the network or the hardware architecture. For example, in [14] it is proposed that the use of heterogeneous networks will positively affect the network power consumption under certain conditions. Cui et al. [4] show how adjusting the number of antennas affects power consumption. In contrast to design changes, operating approaches manipulate the functionality of a BS 
during operation. Here, proposed techniques are the reduction of transmission power [5], the deactivation of unneeded antennas [6], the adaptation of the transmission bandwidth [7] and the use of low power consumption sleep modes of varied durations [8].

The model presented in this paper encompasses all of these approaches to allow for a direct comparison while abstracting parameters which can either be assumed to be constant or have been shown to have little effect in the studied scenarios, such as modulation and coding settings, equipment manufacturing details and leakage powers [13]. To this extent, the following is covered in the proposed model:

- The different BS types of a heterogeneous network are modelled by applying different parameter sets to the same model equations.

- The number of transmission antennas and radio chains affects consumption during design and operation.

- The same holds true for transmission power, which affects the design indirectly by choice of a suitable power amplifier as well as the operation directly.

- Also, transmission bandwidth and sleep modes are modelled in their effect on BS power consumption.

\section{Base Station Power Model}

It was found in [1] that the supply power consumption of a BS can be approximated as an affine function of transmission power. In other words, the consumption can be represented by a static (load-independent) share, $P_{0}$, with an added load-dependent share that increases linearly by a power gradient, $\Delta_{\mathrm{p}}$. The maximum supply power consumption, $P_{1}$, is reached when transmitting at maximum total transmission power, $P_{\max }$. Furthermore, a BS may enter a sleep mode with lowered consumption, $P_{\text {sleep }}$, when it is not transmitting. Fig. 1 shows an illustration. Total power consumption considering the number of sectors, $M_{\mathrm{sec}}$, is then formulated as

$$
P_{\text {supply }}(\chi)= \begin{cases}M_{\text {sec }}\left(P_{1}+\Delta_{\mathrm{p}} P_{\max }(\chi-1)\right) & \text { if } 0<\chi \leq 1 \\ M_{\text {sec }} P_{\text {sleep }} & \text { if } \chi=0,\end{cases}
$$

where $P_{1}=P_{0}+\Delta_{\mathrm{p}} P_{\max }$. The scaling parameter $\chi$ is the load share, where $\chi=1$ indicates a fully loaded system, e.g. transmitting at full power and full 


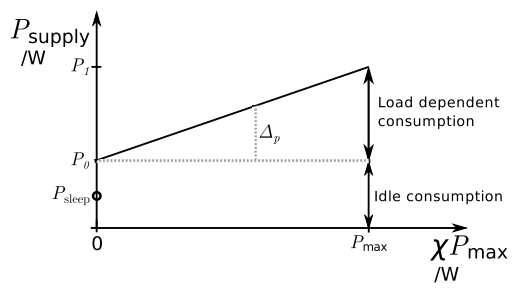

Figure 1: Load-dependent power model for an LTE BS.

bandwidth, and $\chi=0$ indicates an idle system. To further understand the contribution of different parameters on this basic model, we parameterize the maximum supply power consumption, $P_{1}$. We first establish how power consumption scales with the transmission bandwidth in $\mathrm{Hz}, W$, the number of $\mathrm{BS}$ radio chains/antennas, $D$, and the maximum transmission power in $\mathrm{W}$, $P_{\max }$. This requires to consider the main units of a BS: PA, radio frequency (RF) small-signal transceiver, baseband (BB) engine, direct-current (DC)DC converter, active cooling and mains supply (MS). The dependence of the BS units on $W, D$ and $P_{\max }$ can be approximated as follows [13]:

- Both the power consumptions of $\mathrm{BB}$ and $\mathrm{RF}, P_{\mathrm{BB}}$ and $P_{\mathrm{RF}}$, respectively, scale linearly with bandwidth, $W$, in and the number of BS antennas $D$. For some basic consumptions, $P_{\mathrm{BB}}^{\prime}$ and $P_{\mathrm{RF}}^{\prime}$, we thus define

$$
P_{\mathrm{BB}}=D \frac{W}{10 \mathrm{MHz}} P_{\mathrm{BB}}^{\prime}
$$

and

$$
P_{\mathrm{RF}}=D \frac{W}{10 \mathrm{MHz}} P_{\mathrm{RF}}^{\prime}
$$

- The PA power consumption $P_{\mathrm{PA}}$ depends on the maximum transmission power per antenna $P_{\max } / D$ and the PA efficiency $\eta_{\mathrm{PA}}$. Also, possible feeder cable losses, $\sigma_{\text {feed }}$, have to be accounted for:

$$
P_{\mathrm{PA}}=\frac{P_{\mathrm{max}}}{D \eta_{\mathrm{PA}}\left(1-\sigma_{\text {feed }}\right)} \text {. }
$$

- Losses incurred by DC-DC conversion, MS and active cooling scale linearly with the power consumption of other components and may be approximated by the loss factors $\sigma_{\mathrm{DC}}, \sigma_{\mathrm{MS}}$, and $\sigma_{\mathrm{cool}}$, respectively. These losses are included in the model as losses of a total according to [11. Active cooling is typically only applied in Macro type BSs. 
These assumptions are combined to calculate the maximum power consumption of a BS sector,

$$
\begin{aligned}
P_{1} & =\frac{P_{\mathrm{BB}}+P_{\mathrm{RF}}+P_{\mathrm{PA}}}{\left(1-\sigma_{\mathrm{DC}}\right)\left(1-\sigma_{\mathrm{MS}}\right)\left(1-\sigma_{\mathrm{cool}}\right)} \\
& =\frac{D \frac{W}{10 \mathrm{MHz}}\left(P_{\mathrm{BB}}^{\prime}+P_{\mathrm{RF}}^{\prime}\right)+\frac{P_{\max }}{D \eta_{\mathrm{PA}}\left(1-\sigma_{\mathrm{feed}}\right)}}{\left(1-\sigma_{\mathrm{DC}}\right)\left(1-\sigma_{\mathrm{MS}}\right)\left(1-\sigma_{\mathrm{cool}}\right)} .
\end{aligned}
$$

An important characteristic of a PA is that operation at lower transmit powers reduces the efficiency of the PA and that, consequently, power consumption is not a linear function of the PA output power. This is resolved by taking into account the ratio of maximum transmission power of the PA from the data sheet, $P_{\mathrm{PA} \text {,limit }}$ to the maximum transmission power of the PA during operation $\frac{P_{\max }}{D}$. The current transmission power can be adjusted by adapting the DC supply voltage, which impacts the offset power of the PA. The efficiency is assumed to decrease by a factor of $\gamma$ for each halving of the transmission power. The efficiency is thus maximal when $P_{\max }=P_{\mathrm{PA} \text {,limit }}$ in single antenna transmission and was heuristically found to be well-described by

$$
\eta_{\mathrm{PA}}=\eta_{\mathrm{PA}, \max }\left[1-\gamma \log _{2}\left(\frac{P_{\mathrm{PA}, \text { limit }}}{P_{\max } / D}\right)\right],
$$

where $\eta_{\mathrm{PA} \text {,max }}$ is the maximum PA efficiency.

The reduction of power consumption during sleep modes is achieved by powering off PAs and reduced computations necessary in the $\mathrm{BB}$ engine. For simplicity, we only model the dependence on $D$ as each PA is powered off. Thus, $P_{\text {sleep }}$, is approximated as

$$
P_{\text {sleep }}=D P_{\text {sleep }, 0}
$$

where $P_{\text {sleep }, 0}$ is a reference value for the single antenna BS chosen such that $P_{\text {sleep }}$ matches the complex model value for two antennas.

\section{Results and Discussion}

The parameterized power model is applied to approximate the consumption of the Macro, Pico and Femto BSs which are described in the complex model. Parameters are chosen where possible according to [11, such as losses, efficiencies and power limits. The remaining parameters are adapted such that a closer match to the complex model could be achieved. The resulting parameter breakdown is provided in Table1. The proposed and the 


\begin{tabular}{|c|c|c|c|c|c|c|c|c|c|c|c|c|}
\hline BS type & $\begin{array}{c}P_{\text {PA,limit }} \\
/ \mathrm{W}\end{array}$ & $\eta_{\mathrm{PA}, \mathrm{max}}$ & $\gamma$ & $\begin{array}{c}P_{\mathrm{BB}}^{\prime} \\
/ \mathrm{W}\end{array}$ & $\begin{array}{c}P_{\mathrm{RF}}^{\prime} \\
/ \mathrm{W}\end{array}$ & $\sigma_{\text {feed }}$ & $\sigma_{\mathrm{DC}}$ & $\sigma_{\text {cool }}$ & $\sigma_{\mathrm{MS}}$ & $M_{\mathrm{Sec}}$ & $\begin{array}{c}P_{\max } \\
/ \mathrm{W}\end{array}$ & $\begin{array}{c}P_{1} \\
/ \mathrm{W}\end{array}$ \\
\hline Macro & 80.00 & 0.36 & 0.15 & 29.4 & 12.9 & 0.5 & 0.075 & 0.1 & 0.09 & 3 & 40.00 & 460.4 \\
\hline Pico & 0.25 & 0.08 & 0.20 & 4.0 & 1.2 & 0.0 & 0.09 & 0.0 & 0.11 & 1 & 0.25 & 17.4 \\
\hline Femto & 0.10 & 0.05 & 0.10 & 2.5 & 0.6 & 0.0 & 0.09 & 0.0 & 0.11 & 1 & 0.10 & 12.0 \\
\hline
\end{tabular}

Table 1: Parameter breakdown.

complex power models are compared for a bandwidth sweep with a varying number of transmit antennas in Fig. 2, Fig. 3, and Fig. 4, for a Macro, a Pico and a Femto station, respectively. Although two parameters, the bandwidth and the number of BS antennas, are varied, the parameterized model can be seen to closely approximate the complex model for all BS types. The largest deviation of the parameterized model from the complex model occurs when modeling four transmit antennas. This is caused by the fact that the parameterized model considers a constant slope, $\Delta_{\mathrm{p}}$, which is independent of $D$. In contrast, the PA efficiency in the complex model decreases with rising $D$, leading to an increasing slope which can not be matched by a constant slope. This deviation is a trade-off between simplicity and model accuracy.

In addition to providing a solid reference, the model and the parameters can provide a basis for exploration. Individual parameters can be changed to observe the resulting variation in power consumption. With regard to the number of antennas, the parameterized model can only be verified up to four antennas, which is the extent of the complex model. Extending the system bandwidth, for example to $20 \mathrm{MHz}$, is expected to increase the $\mathrm{BB}$ and $\mathrm{RF}$ power consumption. The other parameters such as the transmission power and losses are expected to remain unaffected by different system bandwidths. Adapting the design maximum transmission power, $P_{\max }$, affects the PA efficiencies, which decrease with $P_{\max }$.

\section{Conclusion and remarks}

In this paper, we have provided a parameterized power model which allows calculating the power consumption of a modern BS based on important design and operation parameters. The model is much simpler and more applicable than the model it was derived from. A comparison of the parameterized model with the source model is provided. 


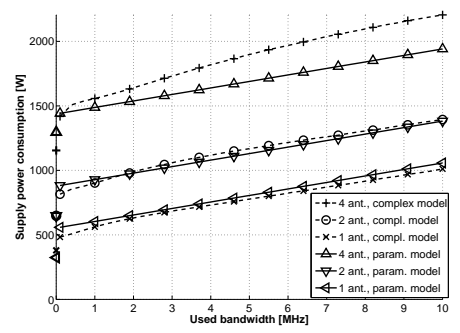

Figure 2: Comparison of the parameterized with the complex model [13] power models for the Macro BS type with $40 \mathrm{~W}$ transmission power.

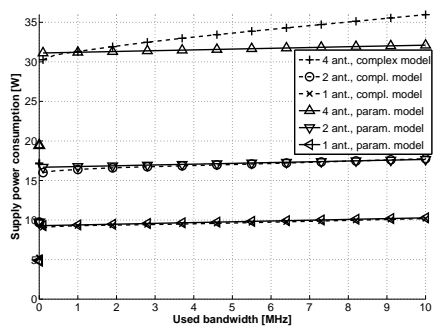

Figure 3: Comparison of the parameterized with the complex model [13] power models for the Pico BS type with $0.25 \mathrm{~W}$ transmission power.

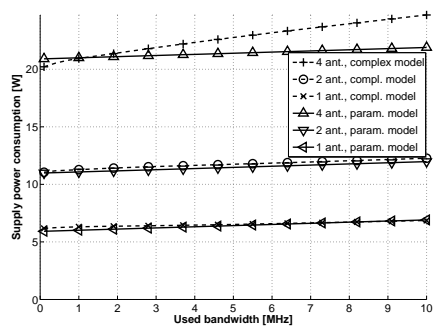

Figure 4: Comparison of the parameterized with the complex model [13] power models for the Femto BS type with $0.1 \mathrm{~W}$ transmission power. 


\section{References}

[1] 3GPP TR 36.927, "Evolved Universal Terrestrial Radio Access (EUTRA); Potential solutions for energy saving for E-UTRAN," Sep. 2012.

[2] A. Fehske, J. Malmodin, G. Biczók, and G. Fettweis, "The Global Carbon Footprint of Mobile Communications - The Ecological and Economic Perspective," IEEE Communications Magazine, 2010.

[3] H. Claussen, L. Ho, and F. Pivit, "Effects of Joint Macrocell and Residential Picocell Deployment on the Network Energy Efficiency," in PIMRC 2008, 2008, pp. 1-6.

[4] S. Cui, A. J. Goldsmith, and A. Bahai, "Energy-Efficiency of MIMO and Cooperative MIMO Techniques in Sensor Networks," IEEE Journal on Selected Areas in Communications, 2004.

[5] H. Holtkamp, G. Auer, and H. Haas, "On Minimizing Base Station Power Consumption," in Proceedings of the IEEE VTC 2011-Fall, 2011.

[6] Z. Xu, C. Yang, G. Y. Li, S. Zhang, Y. Chen, and S. Xu, "EnergyEfficient MIMO-OFDMA Systems based on Switching Off RF Chains," in Proceedings of the VTC Fall 2011, 2011.

[7] A. Ambrosy, M. Wilhelm, O. Blume, and W. Wajda, "Dynamic Bandwidth Management for Energy Savings in Wireless Base Stations," in Proceedings of Globecom 2012, 2012.

[8] P. Frenger, P. Moberg, J. Malmodin, Y. Jading, and I. Gódor, "Reducing Energy Consumption in LTE with Cell DTX," in Proceedings of the IEEE VTC 2011-Spring, 2011.

[9] M. Deruyck, W. Joseph, and L. Martens, "Power Consumption Model for Macrocell and Microcell Base Stations," Transactions on Emerging Telecommunications Technologies, pp. n/a-n/a, 2012.

[10] O. Arnold, F. Richter, G. Fettweis, and O. Blume, "Power Consumption Modeling of Different Base Station Types in Heterogeneous Cellular Networks," in Future Network and Mobile Summit, 2010.

[11] Gunther Auer et al., "How Much Energy is Needed to Run a Wireless Network?" IEEE Wireless Communications, vol. 18, no. 5, pp. $40-49$, Oct 2011. 
[12] G. Auer, V. Giannini, I. Gódor, P. Skillermark, M. Olsson, M. Imran, D. Sabella, M. J. Gonzalez, and C. Desset, "Cellular Energy Efficiency Evaluation Framework," in Proceedings of the VTC 2011-Spring, 2011.

[13] Claude Desset et al., "Flexible Power Modeling of LTE Base Stations," in IEEE WCNC 2012, 2012.

[14] A. Fehske, F. Richter, and G. Fettweis, "Energy efficiency improvements through micro sites in cellular mobile radio networks," in GLOBECOM Workshops, 2009 IEEE, 30 2009-dec. 4 2009, pp. 1 -5. 